\title{
Validación del cuestionario sobre hábitos de consumo de videojuegos en chilenos millennials ${ }^{*}$
}

\author{
Esteban Saavedra Vallejos ${ }^{* *}$ \\ Recibido: 2019-05-22 • Enviado a pares: 2019-06-28 \\ Aprobado por pares: 2019-07-13 - Aceptado: 2019-09-20 \\ https://doi.org/10.22395/angr.v18n36a3
}

\begin{abstract}
Resumen
La masificación del uso de videojuegos ha ido creciendo rápidamente y además, las proyecciones apuntan a que el uso seguirá aumentando en el tiempo. Para la medición del consumo de videojuegos existen numerosos cuestionarios, que en su mayoría se asocian al diagnóstico de trastornos psicológicos. Como el objetivo es medir el uso y consumo de videojuegos y no el diagnóstico de alteraciones psicológicas, se optó por validar el cuestionario de hábitos de consumo de videojuegos, originalmente creado para población española. Por este motivo, se realizó un análisis factorial confirmatorio para población chilena millennials, personas nacidas entre 1980 y 2000. Para la validación, se utilizó una muestra compuesta por cuatrocientos doce estudiantes universitarios. Se calculó la fiabilidad y validez, esta se midió con el coeficiente de fiabilidad compuesta, varianza media extraída y $\alpha$ de Cronbach. La estimación de máxima verosimilitud se evalúo con la corrección robusta de Satorra-Bentler. Los resultados obtenidos para validar la escala muestran que es válida y fiable para la utilización en jóvenes chilenos pertenecientes a la generación millennial o generación $Y$.
\end{abstract}

Palabras clave: Chile; consumo; videojuego; encuesta; análisis factorial.

Artículo de investigación resultado del trabajo final para optar por el título de magíster en Gestión de la Actividad Física y Deporte de la Universidad de Valencia, España.

** Magíster en Dirección y Gestión de la Actividad Física y Deporte, Universidad de Valencia, España. Estudiante de Doctorado en Educación, Universidad Católica de Temuco, Santiago, Chile. Profesor de Educación Física, Universidad de la Frontera, Chile. Correo electrónico: e.saavedra.vallejos@gmail.com. Orcid: https://orcid.org/0000-0002-9220-4320 


\title{
Videogame Consumption Habits Questionnaire Validation in Chilean Millennials
}

\begin{abstract}
The massification of the use of videogames has been growing rapidly and in addition, the projections suggest that use will continue to increase in the future. For the measurement of video game consumption there are numerous questionnaires, which are mostly associated with the diagnosis of psychological disorders. As the objective is to measure the use and consumption of videogames and not the diagnosis of psychological disorders, it was decided to validate the questionnaire of habits of videogame consumption, originally created for the Spanish population. For this reason, a confirmatory factor analysis was carried out for the Chilean millennial population, people born between 1980 and 2000. For the validation, a sample composed of four hundred twelve university students was used. Reliability and validity were calculated with the composite reliability coefficient, mean variance extracted and Cronbach's $\alpha$. The maximum likelihood was estimated with the robust Satorra-Bentler correction. The results obtained to validate the scale show that it is valid and reliable for use in young Chileans belonging to the millennial generation or generation $\mathrm{Y}$.
\end{abstract}

Keywords: Chile; consumption; videogame; survey; factorial analysis.

\section{Validação do questionário sobre hábitos de consumo de videogames nos chilenos millennials}

\begin{abstract}
Resumo
A massificação do uso de videogames vem crescendo com rapidez e, além disso, as projeções apontam a que o uso continuará aumentando. Para medir o consumo de videogames, existem inúmeros questionários, os quais, em sua maioria, estão associados ao diagnóstico de transtornos psicológicos. O objetivo deste artigo é medir o uso e consumo de videogames, e não fazer o diagnóstico de alterações psicológicas, por isso se optou por validar o questionário de hábitos de consumo de videogames, originalmente, criado para a população espanhola. Por esse motivo, foi realizada uma análise fatorial confirmatória para a população chilena millennials, pessoas nascidas entre 1980 e 2000. Para a validação, foi utilizada uma amostra composta por quatrocentos e doze estudantes universitários. Foram calculadas a fiabilidade e a validade; esta foi medida com o coeficiente de confiabilidade composta, variância média extraída e alpha de Cronbach. A estimativa de máxima verossimilhança foi avaliada com a correção robusta de Satorra-Bentler. Os resultados obtidos para validar a escala mostram que é válida e confiável para utilizar em jovens chilenos pertencentes à geração millennial ou à geração $\mathrm{Y}$.
\end{abstract}

Palavras-chave: Chile; consumo; videogame; questionário; análise fatorial. 


\section{Introducción}

El objetivo de esta medición es la validación del cuestionario de hábitos de consumo de videojuegos para población chilena por medio de un análisis factorial exploratorio y confirmatorio. El objetivo es que los investigadores que trabajan en contexto chileno dispongan de un instrumento de medición del consumo de videojuegos de la población millenial. Esta medición puede ser útil para investigadores de áreas ajenas a la psiquiatría, psicología u otra especialidad de medicina afín; investigadores que no deseen diagnosticar enfermedades psicológicas relacionadas con el consumo de videojuegos, sino que tengan un interés en conocer cuánto es el consumo de videojuegos de su muestra. Asimismo, ser utilizado como información que permita la toma de decisiones en materias diversas como educación, economía, marketing, publicidad, cultura, entre muchas otras. También puede ser usada como una variable independiente o dependiente en futuras investigaciones que deseen relacionar el consumo de videojuegos con otra variable.

Para alcanzar el objetivo de la investigación se presenta el contexto de lo que representan hoy los videojuegos (v) (Fitzpatrick, Burkhalter, y Asbridge, 2019; Pinel et al., 2016; Vega y Levy, 2001); la visión positiva y negativa de los vj (Fitzpatrick et al., 2019; Gonzálvez, Espada, y Tejeiro, 2016; Lanz, Bohorodzaner, y Kampfner, 2010; Vega y Levy, 2001); una revisión de la literatura sobre los distintos cuestionarios validados relacionados con el consumo de vi (Andreassen et al., 2017; López Becerra, 2012; Lemmens, Valkenburg, y Peter, 2009; Pontes, Király, Demetrovics, y Griffiths, 2014; Vorderer y Bryant, 2012); el aporte que genera esta validación; y la metodología del estudio presentando los participantes, procedimientos, instrumento y análisis de datos. Luego se muestran los resultados, discusión, conclusión y recomendaciones de uso en futuras investigaciones en esta área.

Para comenzar este artículo, es necesario señalar que los videojuegos llaman la atención de niños, adultos y sobre todo jóvenes debido a su contenido llamativo y motivador (Pinel et al., 2016). Por consiguiente, la industria de los videojuegos crece anualmente en usuarios e ingresos que llegan a los 108,9 billones de dólares en el mundo, con pronósticos de constante crecimiento cada año (Newzoo, 2017). Debido al interés y el crecimiento de la industria de los vJ, se deben tener en cuenta las consecuencias positivas y negativas que conlleva el consumo de vJ.

Por una parte, dentro de los aspectos positivos de los vJ, Vega y Levy (2001) evidencian el desarrollo de habilidades y destrezas sociales que ayudan en el proceso de enseñanza-aprendizaje, específicamente: se da un incremento en la capacidad de planear e imaginar para anticiparse a conflictos; favorece poder dar una respuesta consciente y procesada a los acontecimientos; mejora la autorregulación emocional; 
optimiza la capacidad de resolver conflictos; promueve la socialización y diálogos horizontales sin jerarquías.

Por otra parte, están las evidencias que constatan los perjuicios del uso de videojuegos sedentarios, como lo demuestran Fitzpatrick, Burkhalter y Asbridge (2019), entre sus resultados muestran que cada hora de consumo de vı se asocia a la disminución significativa en la conexión escolar, autoestima y logros académicos. Asimismo, desde el área de la salud se plantea el problema de los vi sedentarios y su participación directa en el fomento del sedentarismo en la población. Este es uno de los factores de riesgo que más influyen en el desarrollo de patologías metabólicas, respiratorias y circulatorias (Uddin y Khan, 2019). Particularmente en Chile, este problema preocupa a las autoridades debido a que el $83 \%$ de los hombres y el $90 \%$ de mujeres son sedentarios y esta realidad trae como consecuencia el aumento del sobrepeso y obesidad en todos los niveles sociales del país (Ministerio de Salud, 2018).

En el ámbito psicológico, los estudios de Gonzálvez, Espada, y Tejeiro (2016) evidencian que el uso problemático de videojuegos produce ansiedad y depresión. Además de la violencia que generan los vj violentos en los jóvenes (Lanz et al., 2010).

Más allá de los aspectos positivos y negativos de los videojuegos, existen estudios en contexto chileno que tratan distintas aristas del tema de los vJ, estos son: marketing en los vı y la industria del entretenimiento (Urzua y Rojas, 2017), análisis del enfoque legislativo (Fajardo y Andrés, 2017), estudios culturales, la ludología, estudios narratológicos, la teoría de diseño de juegos y la semiótica de los vi (Pereira-Henríquez y Alonzo-Zúñiga, 2017), análisis de tendencias temáticas de los estudios sobre vj en Chile, América Latina y España relativos a vı y educación, la relación con las teorías sociales y el aspecto narrativo de estos (Rossi, 2018).

Debido a la atención y preocupación que suscitan los vı, es relevante la validación de un instrumento que mida el consumo de videojuegos en Chile con el fin de avanzar en el conocimiento contextualizado en esta área. Por ello, se realizó una búsqueda de escalas de medición para el consumo de videojuegos, esta tuvo resultados tales como: video game uses and gratifications as predictors of use and game preference (Vorderer y Bryant, 2012), que mide los motivos que tienen las personas para jugar vj; adicción a internet, redes sociales y videojuegos, escala que tiene por objetivo principal medir la relación entre el uso adictivo de las redes sociales, vı y síntomas de trastornos psiquiátricos (Andreassen et al., 2017); game addiction scale for adolescents (GASA) (Lemmens et al., 2009), escala que mide la adicción a los videojuegos por parte de los adolecentes; internet gaming disorder (IGD20) (Pontes et al., 2014) mide el nivel de adicción a los vı en línea o jugados en internet. Estos resultados de búsqueda muestran una tendencia a la medición del uso excesivo de vi y diagnóstico de trastornos adictivos. Como el objetivo de este estudio es medir el uso de videojuegos de los jóvenes sin tener un sesgo relacionado con patologías 
psicológicas o psiquiatras, se optó por validar en contexto chileno el cuestionario de hábitos de consumo de videojuegos (López Becerra, 2012) creado para población española.

Los aportes de esta investigación son tres: 1) comprobar y diferenciar el consumo de vj entre mujeres y hombres; 2) proveer a la comunidad científica local un instrumento validado para la medición del consumo de videojuegos; 3) el cuestionario original creado en España solo utiliza como criterio de validez de constructo el análisis factorial exploratorio (AFE), en la presente validación, además de hacer el AFE se realiza un análisis factorial confirmatorio (AFC) para dar una validez y fiabilidad mayor al constructo, puesto que el AFE se utiliza para definir constructos y el AFC para demostrar la validez de constructo de la definición hecha por el AfE (Pérez-Gil, Chacón, y Moreno, 2000).

\section{Método}

\section{Participantes}

La muestra está constituida por estudiantes de pregrado y posgrado de las áreas de las Facultad de Educación, Ciencias Sociales y Humanidades, Facultad de Ingeniería y Ciencias, Facultad de Medicina, Facultad de Ciencias Jurídicas y Empresariales de tres instituciones de educación superior de la ciudad de Temuco, Chile. Para ello se analizó una población de 412 estudiantes. Del total de encuestados, 171 son hombres $(41,5 \%$ ) y 241 son mujeres (58,8\%), la media de edad es de 22,72 (DT=4,66). El criterio de selección de la muestra fue la edad, entre 18 y 37 años de edad, tipificados como la generación Y o millennials (Cekada, 2012).

\section{Procedimiento}

Para la obtención de datos se decidió, en primer lugar, aplicar el cuestionario en línea in situ, esta se podía contestar a través de un smartphone (teléfono inteligente) o computadora conectada a internet, en los diversos cursos y post-títulos de las diversas universidades de Temuco en las que todos los sujetos cumplían con los criterios básicos del estudio anteriormente mencionados.

Para contestar el cuestionario, los estudiantes tardaron entre siete y quince minutos, no se presentó incidencia alguna por demora. El proceso de aplicación se llevó a cabo a partir de abril del 2018 y la información se recolectó hasta junio del 2018.

\section{Instrumento}

Como instrumento de recolección de información se utilizó el Cuestionario de Hábitos de Consumo de Videojuegos (cHcv) (López Becerra, 2012), compuesto por veinticuatro ítems, dividido en dos áreas, una es la escala de medición de hábitos de consumo de 
videojuegos (diecinueve ítems) y otra recolecta datos descriptivos de frecuencia en el uso de videojuegos (disponible en http://goo.gl/itpamA).

\section{Análisis de datos}

El análisis estadístico de los datos se realizó por medio de los paquetes estadísticos SPSS v25 (IBM SPSS, 2016) y EOS 6,3 (Bentler y Wu, 2015). En primer lugar, con la muestra $(n=412)$ se calcularon los estadísticos descriptivos más importantes para cada ítem; a través del método de rotación Varimax con normalización Kaiser se extrajeron tres factores (tabla 1): "grado de atracción y uso de los videojuegos", "interferencia de los videojuegos con otras actividades" y "nivel de inquietud respecto a los videojuegos". Seguidamente, la consistencia interna del instrumento se examinó con el índice alfa de Cronbach, de igual forma que en el estudio original. Sin embargo, este no tiene en cuenta la influencia sobre la fiabilidad del resto de constructos, por esto se calculó el coeficiente de fiabilidad compuesta y la varianza media extraída (Fornell y Larcker, 1981)

\section{Resultados}

\section{Análisis factorial}

Por medio de un análisis factorial exploratorio, a través del método de rotación Varimax con normalización Kaiser se extrajeron tres factores (tabla 1): "grado de atracción y uso de los videojuegos", "interferencia de los videojuegos con otras actividades" y "nivel de inquietud respecto a los videojuegos". Este análisis fue posible dado que se obtuvieron resultados adecuados en la prueba Keiser Mayer Olkin $(0,95)$ y de esfericidad de Barttlet ( $p<0,001$ ), los que se denominaron del mismo modo que en la construcción original de este cuestionario.

El factor 1 "grado de atracción y uso de los videojuegos", da información sobre el grado de atracción y uso de videojuegos. Está formado por los ítems uno a siete y estos explican el 29,77 \% de la varianza total del cuestionario.

El factor 2 "interferencia de los videojuegos con otras actividades", da información sobre el grado de interferencia que tiene jugar videojuegos respecto a otras actividades de la vida diaria tales como estudiar, trabajar y otras actividades recreativas. Está formado por los ítems ocho a once, diecisiete a diecinueve y estos explican el $24,86 \%$ de la varianza total del cuestionario.

El factor 3 "nivel de inquietud respecto a los videojuegos" da información sobre las actividades anexas a jugar, pero que tienen directa relación como hablar con amigos sobre videojuegos, buscar información, visitar tiendas especializadas, entre otras. Está formado por los ítems doce a dieciséis y explican el 17,76 \% de la varianza total del cuestionario. 
La fiabilidad del cuestionario se midió con el coeficiente alpha de Cronbach 0,958. El alfa de los factores 1, 2 y 3 son mayores a 0,89.

Tabla 1. Matriz de componente rotado

\begin{tabular}{|c|c|c|c|c|}
\hline \multirow{2}{*}{ Ítem } & \multicolumn{3}{|c|}{ Componente } & \multirow[b]{2}{*}{ h2 } \\
\hline & 1 & 2 & 3 & \\
\hline 1 & 0,85 & & & 0,83 \\
\hline 2 & 0,76 & & & 0,76 \\
\hline 3 & 0,77 & & & 0,77 \\
\hline 4 & 0,72 & & & 0,73 \\
\hline 5 & 0,79 & & & 0,76 \\
\hline 6 & 0,84 & & & 0,76 \\
\hline 7 & 0,75 & & & 0,68 \\
\hline 8 & & 0,59 & & 0,63 \\
\hline 9 & & 0,63 & & 0,61 \\
\hline 10 & & 0,69 & & 0,66 \\
\hline 11 & & 0,76 & & 0,73 \\
\hline 12 & & & 0,72 & 0,76 \\
\hline 13 & & & 0,55 & 0,66 \\
\hline 14 & & & 0,79 & 0,72 \\
\hline 15 & & & 0,59 & 0,76 \\
\hline 16 & & & 0,73 & 0,70 \\
\hline 17 & & 0,75 & & 0,77 \\
\hline 18 & & 0,79 & & 0,75 \\
\hline 19 & & 0,80 & & 0,72 \\
\hline Autovalor & 10,94 & 1,76 & 1,05 & \\
\hline Alfa de Cronbach & 0,94 & 0,91 & 0,89 & \\
\hline Varianza explicada & 29,77 & 24,86 & 17,76 & \\
\hline Varianza total & & 72,39 & & \\
\hline
\end{tabular}

Fuente: elaboración propia.

En total se analizaron 19 ítems, todos de la escala original. En la tabla 2 se presenta la media, desviación típica y correlación ítem-total. El alfa de Cronbach si se elimina el elemento, equivale al valor de alfa si eliminamos cada uno de los ítems para cada uno de ellos, Asimetría y Curtosis. En general, todos los ítems parecen contribuir de manera adecuada al conjunto de la escala, es decir que presentan una correlación relativamente alta con toda la escala y su fiabilidad total $(\alpha=0,958)$ no mejora al eliminar alguno de ellos. 
Tabla 2. Análisis de diecinueve ítems

\begin{tabular}{|c|c|c|c|c|c|c|}
\hline Ítem & M & $\mathrm{DE}$ & rjx & $(\alpha-x)$ & A & K \\
\hline \multicolumn{7}{|c|}{ Grado de atracción y uso de los videojuegos $\alpha=0,94$} \\
\hline 1 & 3,18 & 1,39 & 0,81 & 0,96 & $-0,12$ & $-1,24$ \\
\hline 2 & 2,49 & 1,32 & 0,82 & 0,96 & 0,50 & $-0,87$ \\
\hline 3 & 2,74 & 1,38 & 0,82 & 0,96 & 0,32 & $-1,10$ \\
\hline 4 & 2,80 & 1,40 & 0,80 & 0,96 & 0,30 & $-1,19$ \\
\hline 5 & 2,55 & 1,31 & 0,79 & 0,96 & 0,37 & $-0,96$ \\
\hline 6 & 3,51 & 1,33 & 0,74 & 0,97 & $-0,43$ & $-1,03$ \\
\hline 7 & 3,33 & 1,48 & 0,72 & 0,97 & $-0,33$ & $-1,28$ \\
\hline \multicolumn{7}{|c|}{ Interferencia de los videojuegos con otras actividades $\alpha=0,91$} \\
\hline 8 & 1,82 & 1,14 & 0,73 & 0,97 & 1,34 & 0,91 \\
\hline 9 & 2,21 & 1,41 & 0,68 & 0,97 & 0,84 & $-0,67$ \\
\hline 10 & 1,45 & 0,90 & 0,61 & 0,97 & 2,23 & 4,69 \\
\hline 11 & 1,62 & 1,05 & 0,69 & 0,97 & 1,75 & 2,32 \\
\hline 17 & 1,65 & 1,09 & 0,74 & 0,97 & 1,70 & 2,02 \\
\hline 18 & 1,55 & 1,01 & 0,68 & 0,97 & 2,00 & 3,26 \\
\hline 19 & 1,67 & 1,09 & 0,64 & 0,97 & 1,75 & 2,25 \\
\hline \multicolumn{7}{|c|}{ Nivel de inquietud respecto a los videojuegos $\alpha=0,89$} \\
\hline 12 & 1,77 & 1,20 & 0,73 & 0,97 & 1,46 & 0,97 \\
\hline 13 & 2,01 & 1,33 & 0,75 & 0,97 & 1,06 & $-0,19$ \\
\hline 14 & 1,29 & 0,76 & 0,54 & 0,97 & 2,88 & 8,24 \\
\hline 15 & 1,92 & 1,21 & 0,81 & 0,96 & 1,12 & 0,11 \\
\hline 16 & 1,54 & 1,00 & 0,65 & 0,97 & 2,01 & 3,40 \\
\hline
\end{tabular}

Nota: media: M; desviación estándar: SD; correlación ítem-total: rjx; alfa de Cronbach si se elimina el elemento: $\alpha$-x; asimetría: A; Curtosis: K.

Fuente: elaboración propia.

La consistencia interna del instrumento de diecinueve ítems se examinó mediante el índice alfa de Cronbach. Sin embargo, este no tiene en cuenta la influencia sobre la fiabilidad del resto de constructos, por esto se calculó el coeficiente de fiabilidad compuesta (CFC) y la varianza media extraída (AvE) (Fornell y Larcker, 1981). El valor mínimo que se considera adecuado para el coeficiente de fiabilidad compuesta es 0,7 (Nunnally, 1978) y para los valores del AvE se recomienda que sean > 0,40 (Aldás, 2000). 
Los valores hallados presentan, en general, una adecuada consistencia interna para la escala, similar a la versión original del instrumento.

En la tabla 3 se presenta la varianza media extraída, coeficiente de fiabilidad compuesta y alfa de Cronbach del cuestionario completo y por factor.

Tabla 3. Fiabilidad compuesta, varianza media extraída y alfa de Cronbach

\begin{tabular}{lccc}
\hline \multicolumn{1}{c}{$\begin{array}{c}\text { Cuestionario completo } \\
(\alpha=0,958)\end{array}$} & $c f c$ & ave & $\alpha$ \\
\hline Grado de atracción y uso de los videojuegos & 0,94 & 0,70 & 0,94 \\
\hline Interferencia de los videojuegos con otras actividades & 0,92 & 0,62 & 0,91 \\
\hline Nivel de inquietud respecto a los videojuegos & 0,89 & 0,62 & 0,89 \\
\hline
\end{tabular}

Fuente: elaboración propia.

La estimación de máxima verosimilitud (ML) con la corrección robusta de SatorraBentler se utilizó en todos los análisis (Bentler, 2006), ya que es el método más sólido de estimación con datos no normales. En lo que respecta a la significación de $\chi^{2}(<0,01)$, en ningún caso se puede garantizar un ajuste adecuado. Sin embargo, como esta estadística está estrechamente relacionada con el tamaño de la muestra, se analizaron otros indicadores, como la relación entre $\chi^{2}$ y sus grados de libertad que son inferiores a cinco, considerados aceptables $(\chi 2 / \mathrm{df}=3,54)$ (Mclver y Carmines, 1981). Los índices de bondad de ajuste como el índice de ajuste comparativo (CFI) y el índice incremental (IFI) (valores superiores a 0,9) indican un ajuste adecuado (MacCallum y Austin, 2000), $\mathrm{CFI}=0,91$, IFI $=0,91$ y el error cuadrático medio de aproximación (RMSEA), en el que los valores por debajo de 0,08 (RMSEA $=0,079)$ son indicadores de un ajuste adecuado (Browne y Cudeck, 1992).

Hubo correlaciones significativas entre el consumo de videojuegos y tiempo dedicado a los videojuegos $(r=0,772, p<0,01)$, frecuencia semanal de consumo de VJ $(r=0,755 p<0,01)$.

Tabla 4. Correlaciones entre variables

\begin{tabular}{lccc}
\hline & Consumo de videojuegos & $\begin{array}{c}\text { Tiempo dedicado } \\
\text { a los videojuegos }\end{array}$ & $\begin{array}{c}\text { Frecuencia semanal } \\
\text { de consumo de VJ }\end{array}$ \\
\hline Consumo de videojuegos & 1 & & \\
\hline Tiempo dedicado a los videojuegos & $0,772^{* *}$ & 1 & 1 \\
\hline Frecuencia semanal de consumo de VJ & $0,755^{* *}$ & $0,783^{* *}$ & \\
\hline " Correlaciones significativas entre el consumo de videojuegos y tiempo dedicado a los videojuegos, frecuencia semanal de \\
consumo de vJ.
\end{tabular}

Fuente: elaboración propia. 


\section{Hábitos de consumo de videojuegos y género}

Al igual que en el estudio de validación del chcv (López Becerra, 2012), se comparan las diferencias de hábitos de consumo por género luego de aplicar la prueba U de Mann-Whitney, puesto que la normalidad de la muestra calculada con el test Kolmogorov-Smirnov da un valor de $p<0,05$. Los resultados indican que son significativos $\mathrm{y}$, por lo tanto, a partir de estos datos se puede afirmar que existe una diferencia significativa entre hombres y mujeres. Además, se detecta que el consumo de videojuegos por parte de los hombres es significativamente mayor al de las mujeres. U de Mann-Whitney $=8244,5$, media (hombres $=272,22$, mujeres $=155,21$ ), $Z=-11,129, p<0,05$.

Tabla 5. Hábitos de consumo de videojuego por género

\begin{tabular}{lr}
\hline \multicolumn{2}{c}{ Estadísticos de prueba } \\
\hline \multicolumn{2}{c}{ Hábitos de consumo de videojuegos } \\
\hline U de Mann-Whitney & $8.244,500$ \\
\hline W de Wilcoxon & $37.405,500$ \\
\hline$Z$ & $-11,129$ \\
\hline Sig. asintótica(bilateral) & 0,000 \\
\hline \multicolumn{2}{r}{ Fuente: elaboración propia. }
\end{tabular}

\section{Discusión}

Tener un conocimiento sobre cuánto es el consumo de videojuegos de los jóvenes y adultos es importante para la planificación de las actividades diarias de la vida, para poder organizarse y no caer en el consumo excesivo de videojuegos. Por esta razón, se ajusta y valida la escala de hábitos de consumo de videojuegos creada por López Becerra (2012). Se hace el mismo análisis original y adicionalmente, se realiza un análisis factorial confirmatorio para jóvenes millennials chilenos. En relación con la presente investigación se demuestra la confiabilidad de la escala en su conjunto con una consistencia aceptable (alfa de Cronbach 0,958 ) y el análisis factorial confirmatorio avala la validez y fiabilidad de cada ítem.

A diferencia del cuestionario original, el análisis factorial exploratorio, a través del método de rotación Varimax con normalización Kaiser dio como resultado tres factores y no cuatro, como el original (López Becerra, 2012). En esta validación el factor original "interferencia de los videojuegos con las actividades académicas" se fusiona con "interferencia de los videojuegos con otras actividades".

Es importante aclarar que este instrumento de medición es una apreciación subjetiva y propia de cada sujeto sobre su consumo de videojuegos en relación con: 
1) grado de atracción y uso de los videojuegos; 2) interferencia de los videojuegos con otras actividades y 3 ) nivel de inquietud respecto a los videojuegos.

En relación con los tres aportes declarados en la introducción de esta investigación se concluye que: 1) los hombres tienen un grado mayor de atracción y uso de videojuegos y le dedican más tiempo semanal que las mujeres de la misma edad; 2) estadísticamente, esta es una escala fiable para aplicar en jóvenes y adultos chilenos pertenecientes a la generación millennial o generación Y; 3) el análisis factorial confirmatorio comprueba la validez y fiabilidad de cada ítem.

Se sugiere aplicar esta escala de medición de consumo de videojuegos y complementar la información con una aplicación para teléfonos inteligentes, consolas de videojuegos o computadoras personales que miden el tiempo exacto que la persona dedica a jugar videojuegos, debido a que esta escala contempla una apreciación personal y subjetiva del encuestado sobre el tiempo que emplea en jugar videojuegos y no un dato real, como lo puede proveer una aplicación tecnológica como la descrita.

Las investigaciones futuras sobre videojuegos se deberían centrar en averiguar los beneficios y modos de aprovechamiento positivo de los videojuegos en ámbitos como la educación, promoción de valores, aumento del consumo energético diario con los videojuegos activos; más que cuantificar el daño en la salud mental y física de las personas debido a que la literatura abunda en estas últimas dos temáticas. Asimismo, se propone una actualización de las preguntas en relación con el medio de consumo de videojuegos, diferenciar si consume videojuegos activos o convencionales y complementar la información con el hábito de consumo del teléfono inteligente e internet en sus diversas plataformas de accesibilidad.

En relación con la labor realizada por los profesionales que trabajamos con personas de este rango etario, nos queda una misión importante y difícil: combatir una de las aristas negativas del consumo de videojuegos, como el uso excesivo que conduce a trastornos psiquiátricos y el sedentarismo de la población al fomentar la promoción de la actividad física; el uso de los videojuegos puede coexistir perfectamente con la práctica física, ya sea por medio de los videojuegos activos o motivando la ejercitación.

Los videojuegos pueden ser una herramienta poderosa para el mejoramiento de la acción educativa y la promoción de la salud, pero también una herramienta generadora de adicciones y traumas. Como toda herramienta, tiene una utilidad buena y mala, depende de nosotros utilizar y privilegiar el uso positivo de los videojuegos en desmedro del uso negativo.

\section{Referencias}

Aldás, J. (2000). Problemas metodológicos de la evaluación de los modelos de exposición: Especial referencia al caso español. En J. Bigné (Ed.) Temas de Planificación de Medios (pp. 89-114). Esic. 
Andreassen, C. S., Billieux, J., Griffiths, M. D., Kuss, D. J., Demetrovics, Z., Mazzoni, E. y Pallesen, S. (2017). Adicción a internet, redes sociales y videojuegos: La relación entre el uso adictivo de las redes sociales y los video juegos y síntomas de trastornos psiquiátricos: un estudio transversal a gran escala. RET: revista de toxicomanías, (81), 2-2. https://dialnet.unirioja.es/servlet/ articulo?codigo $=6390833$

Bentler, P. M. (2006). EQS 6, Structural Equations Program Manual. Multivariate Software Inc. http:// www.econ.upf.edu/ satorra/CourseSEMVienna2010/EOSManual.pdf

Bentler, P., y Wu, E. (2015). Supplement to EQS 6.3 for windows user's guide. Multivariate Software, Inc. http://www.mvsoft.com/pub/eqs/SetupEQS63E.exe

Browne, M. W., y Cudeck, R. (1992). Alternative Ways of Assessing Model Fit. Sociological Methods E Research, 21(2), 230-258. https://doi.org/10.1177/0049124192021002005

Cekada, T. L. (2012). Training a Multigenerational Workforce: Understanding Key Needs \& Learning Styles. Professional Safety, 57(03), 40-44. https://www.onepetro.org/journal-paper/ASSE-12-03-40

Fajardo, P., y Andrés, J. (2017). Prohibido jugar: Análisis de las leyes de videojuegos en Chile y Colombia. Lúdicamente, 6(12). http://ppct.caicyt.gov.ar/index.php/ludicamente/article/ view/10315

Fitzpatrick, C., Burkhalter, R., y Asbridge, M. (2019). Adolescent media use and its association to wellbeing in a Canadian national sample. Preventive Medicine Reports, 14, 6. https://doi. org/10.1016/j.pmedr.2019.100867

Fornell, C., y Larcker, D. F. (1981). Evaluating Structural Equation Models with Unobservable Variables and Measurement Error. Journal of Marketing Research, 18(1), 39-50. https://doi. org/10.2307/3151312

Gonzálvez, M. T., Espada, J. P., y Tejeiro, R. (2016). El uso problemático de videojuegos está relacionado con problemas emocionales en adolescentes. Adicciones, 29(3), 180 185. https:// doi.org/10.20882/adicciones.745

IBM Corp. (2017). IBM SPSS Statistics for Windows (version 25). IBM Corp. https:/www.ibm.com/cl-es/ analytics/academic-statistical-software

Lanz, P. M., Bohorodzaner, S., y Kampfner, E. (2010). Violencia y videojuegos en adolescentes chilenos y mexicanos. Revista Chilena de Neuropsicología, 5(3), 199-206. http://www.redalyc.org/ articulo.oa?id=179318868003

Lemmens, J. S., Valkenburg, P. M. y Peter, J. (2009). Development and Validation of a Game Addiction Scale for Adolescents. Media Psychology, 12(1), 77-95. https://doi. org/10.1080/15213260802669458

López Becerra, F. (2012). Construcción y validación de un cuestionario sobre los hábitos de consumo de videojuegos en preadolescentes. Edutec. Revista Electrónica de Tecnología Educativa, (40), a197-a197. https://www.edutec.es/revista/index.php/edutec-e/article/view/361

MacCallum, R. C. y Austin, J. T. (2000). Applications of structural equation modeling in psychological research. Annual Review of Psychology, 51, 201-226. https://doi.org/10.1146/ annurev.psych.51.1.201 
McIver, J. y Carmines, E. G. (1981). Unidimensional scaling. SAGE.

Ministerio de Salud. (2018). Primeros y segundos resultados de Encuesta nacional de Salud 2016-2017. https://www.minsal.cl/wp-content/uploads/2017/11/ENS-2016-17_PRIMEROS-RESULTADOS. pdf

Newzoo. (2017). Newzoo's Global Games Market Report. https://newzoo.com/solutions/standard/ market-forecasts/global-games-market-report/

Nunnally, J. (1978). Psychometric methods (2 ed.). McGraw-Hill.

Pereira-Henríquez, F., y Alonzo-Zúñiga, T. (2017). Hacia una conceptualización de los videojuegos como discursos multimodales electrónicos. Anagramas Rumbos y Sentidos de la Comunicación, 15(30), 51-64. https://doi.org/10.22395/angr.v15n30a2

Pérez-Gil, J. A., Chacón, S., y Moreno, R. (2000). Validez de constructo: El uso de análisis factorial exploratorio-confirmatorio para obtener evidencias de validez. Psicothema, 12(Su2), 442-446. http://www.psicothema.es/pdf/601.pdf

Pinel, C., Zurita Ortega, F., Espejo Garcés, T., Chacon Cuberos, R., Castro Sanchez, M., y Pérez Cortes, A. (2016). Interaction between frequency of play and healthy habits in Granada' students. Revista Latinoamericana De Tecnologia Educativa-Relatec, 15(2), 169-176. https://doi. org/10.17398/1695-288X.15.2.169

Pontes, H. M., Király, O., Demetrovics, Z., y Griffiths, M. D. (2014). The Conceptualisation and Measurement of DSM-5 Internet Gaming Disorder: The Development of the IGD-20 Test. Plos One, 9(10), el 10137. https://doi.org/10.1371/journal.pone.0110137

Rossi, L. S. R. (2018). Un mapa de los estudios latinoamericanos y españoles sobre videojuegos. Observatorio (OBS*), 12(1). https://doi.org/10.15847/obsOBS12120181170

Uddin, R., y Khan, A. (2019). Sedentary behaviour is associated with overweight and obesity among adolescents: Evidence from a population-based study. Acta Paediatrica, 108(8), 1545 1546. https://doi.org/10.1111/apa.14827

Urzúa, J., y Rojas, C. (2017). Influencias del Marketing digital en la decisión de compra: Modelo ZMOT en el sector de entretenimiento en Chile. International Journal of Management \& Social Studies, 1(2), 49-64.

Vega, A. L. L., y Levy, D. P. C. (2001). Los videojuegos en el contexto de las nuevas tecnologías: Relación entre las actividades lúdicas actuales, la conducta y el aprendizaje. Píxel-Bit. Revista de Medios y Educación, O(17), 33-45. https://recyt.fecyt.es/index.php/pixel/article/view/61 175

Vorderer, P., y Bryant, J. (eds.) (2012). Playing Video Games: Motives, Responses, and Consequences. Routledge. https://doi.org/10.4324/9780203873700 